\title{
The Assignment of Four New Loci, Including the Coumarin Sensitivity Locus couA, to Linkage Group VII of Dictyostelium discoideum
}

\author{
By D. L. WELKER*† AND K. L. WILLIAMS† \\ Department of Genetics, Research School of Biological Sciences, \\ The Australian National University, P.O. Box 475, Canberra City 2601, Australia
}

(Received 27 November 1979; revised 4 March 1980)

\begin{abstract}
The assignment to linkage group VII of the coumarin sensitivity mutation couA351, which leads to a loss of colony-forming ability on agar containing coumarin, was established using the linkage group VII markers cobAl and tsgK21. Complementation of the couA35I and $b s g A 5$ mutations was an effective method of selecting heterozygous diploids at $21 \pm 1{ }^{\circ} \mathrm{C}$, i.e. without requiring a temperature sensitivity mutation. A morphological mutation, frtB353, which affects the distribution of fruiting bodies was also assigned to linkage group VII. Both the couA351 and frtB353 mutations were discovered in the $t s g K 21$ strain NP187. Eight independently isolated, recessive mutations leading to resistance to $300 \mu \mathrm{g} \mathrm{CoCl}_{2} \mathrm{ml}^{-1}$ were allelic with the cobAl mutation. The partially dominant cob-353 mutation was shown to map in linkage group VII on the basis of its segregation relative to the couA351 and tsgK21 mutations and is almost certainly an allele of the $\operatorname{cobA}$ locus. These results are consistent with there being only a single locus at which mutations can lead to resistance to high concentrations of $\mathrm{CoCl}_{2}$. Two temperature sensitivity mutations tsgM357 and tsgG4 were also assigned to linkage group VII.
\end{abstract}

\section{INTRODUCTION}

The cellular slime mould Dictyostelium discoideum has been the subject of numerous studies since, on starvation, the uninucleate amoebae aggregate and proceed through a developmental cycle in which the aggregate differentiates into a fruiting body consisting of only three cell types (Loomis, 1975). With the establishment of genetic analysis based on the parasexual cycle, combined genetic and biochemical studies of the developmental cycle of $D$. discoideum can be undertaken. The parasexual cycle requires selective procedures to obtain diploids that result from the fusion of pairs of parental haploids and to obtain segregant haploids from these diploids in which the linkage groups of the parental haploids assort independently (Newell, 1978; Williams, 1978; Williams \& Barrand, 1978). Genetic analysis is also dependent on the availability of useful mutations to serve as markers for the linkage groups to enable the identification of haploid segregants with particular genotypes. Drug sensitivity mutations, such as the couA351 mutation which we describe here, have so far played only a small role in genetic analysis of $D$. discoideum but are potentially useful in selection of heterozygous diploids and as genetic markers.

To date, six linkage groups have been identified in $D$. discoideum, although the karyotype contains seven chromosomes (Robson \& Williams, 1977; Zada-Hames, 1977). Relatively few genetic loci have been assigned to linkage group VII (Williams \& Newell, 1976; Ratner

† Present address: Max Planck Institut für Biochemie, D-8033 Martinsried bei München, F.R.G. 
\& Newell, 1978; Ross \& Newell, 1979) and the availability of further genetic markers for this linkage group would be useful. In this report, we describe mutations which map at four additional loci on linkage group VII and also nine independently isolated cobalt resistance mutations, all of which map at the $\operatorname{cobA}$ locus of linkage group VII.

\section{METHODS}

Growth of amoebae. Amoebae were grown on a cobalt-resistant strain of Klebsiella aerogenes at $21 \pm 1{ }^{\circ} \mathrm{C}$ on SM-agar (Sussman, 1966) or SM-agar containing inhibitors prepared as described previously (Williams, 1978; Williams \& Newell, 1976). Medium containing coumarin was prepared by adding coumarin prior to sterilization. The concentrations of coumarin used were not toxic to $K$. aerogenes or Bacillus subtilis. Stocks were cloned weekly or stored for long periods on silica gel at $4^{\circ} \mathrm{C}$.

Strains used. All strains of $D$. discoideum used in this work were derived from the NC4 wild isolate and the haploid strains used are described in Table 1.

Selection of diploids. Haploid amoebae were co-aggregated in $20 \mathrm{mM}-\mathrm{CaCl}_{2}$ with shaking in either Falcon 3040 dishes (Williams \& Newell, 1976) for routine crosses, or in Erlenmeyer flasks (Welker \& Deering, 1978) for quantitative experiments. Diploids were isolated either on the basis of complementation of non-allelic recessive growth mutations (Loomis, 1969; Newell et al., 1977) or the complementation of a recessive temperature sensitivity mutation and the dominance of a mutation leading to resistance to $\mathrm{CoCl}_{2}$ of the same parental haploid (Williams, 1978).

Haploidization. Haploid segregants were obtained from diploids plated clonally on SM-agar plates that contained the haploidizing agent ben late at between 20 and $50 \mu \mathrm{g} \mathrm{ml}^{-1}$ (Williams \& Barrand, 1978).

Characterization of segregants. The phenotypes for most characteristics were scored on appropriate media, as described previously (Williams et al., 1974; Williams, 1978; Newell et al., 1977; Free et al., 1976), using either toothpicked cells or cell suspensions (Welker \& Deering, 1976, 1978). In particular, the bsg $A 5$ and couA35I mutations were scored more easily and mixed clones were more readily apparent using the suspension technique. Due to the increase in toxicity with distance from the edge of SM-agar plates containing $\mathrm{CoCl}_{2}$, resistance to $\mathrm{CoCl}_{2}$ was scored with cells toothpicked 5 to $10 \mathrm{~mm}$ from the edge of the Petri dish (Williams, 1978). Coumarin sensitivity was scored on SM-agar plates containing either 1.0 or 1.3 mM-coumarin; usually segregants were tested on both coumarin concentrations. Spore sizes and shapes were determined by microscopic examination at $400 \times$ magnification.

Origin of mutations. Mutants carrying the new $\mathrm{CoCl}_{2}$ resistance mutations described in this paper were probably spontaneous since they were selected by plating unmutagenized amoebae on SM-agar plates containing between 300 and $400 \mu \mathrm{g} \mathrm{CoCl}_{2} \mathrm{ml}^{-1}$. The temperature sensitivity mutation $t \mathrm{sgG} 4$ was found by Rothman \& Alexander (1975) after mutagenesis with $N$-methyl- $N^{\prime}$-nitro- $N$-nitrosoguanidine (MNNG). The tsgM357 mutation was obtained in this laboratory following MNNG mutagenesis of strain HU526 (1 to

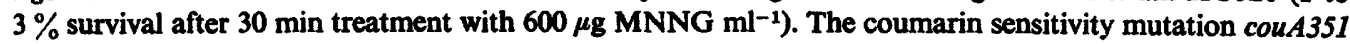
and the morphological mutation frtB353 were discovered in this laboratory in strain NP187 and are likely to have been induced during the mutagenesis of strain NP20 to obtain the tsgK21 mutation of NP187 (P. C. Newell, unpublished results).

Chemicals. Coumarin (1,2-benzopyrone) and cycloheximide were purchased from Sigma. Ben late was from Dupont (New South Wales agents), while methanol and $\mathrm{CoCl}_{\mathbf{2}}$ were from May \& Baker (analytical reagent grade).

\section{RESULTS}

\section{A coumarin sensitivity locus on linkage group VII}

While searching for new types of mutations of use as genetic markers for $D$. discoideum, we discovered one which led to an increased sensitivity to the drug coumarin. This mutation, couA351, is present in strain NP187, but not in strain NP20 from which NP187 was derived.

As shown in Fig. 1, the colony-forming ability of amoebae bearing the couA351 mutation dramatically decreased when the amoebae were plated on SM-agar plates containing coumarin at concentrations above $0.5 \mathrm{mM}$. The parental strain NP20, and other wild-type strains tested, such as XP99, grew more slowly but showed little or no loss of colonyforming ability when plated on media containing up to 1.3 mM-coumarin. The couA351 mutation is recessive since the diploid DU584, which was constructed by fusion of the 


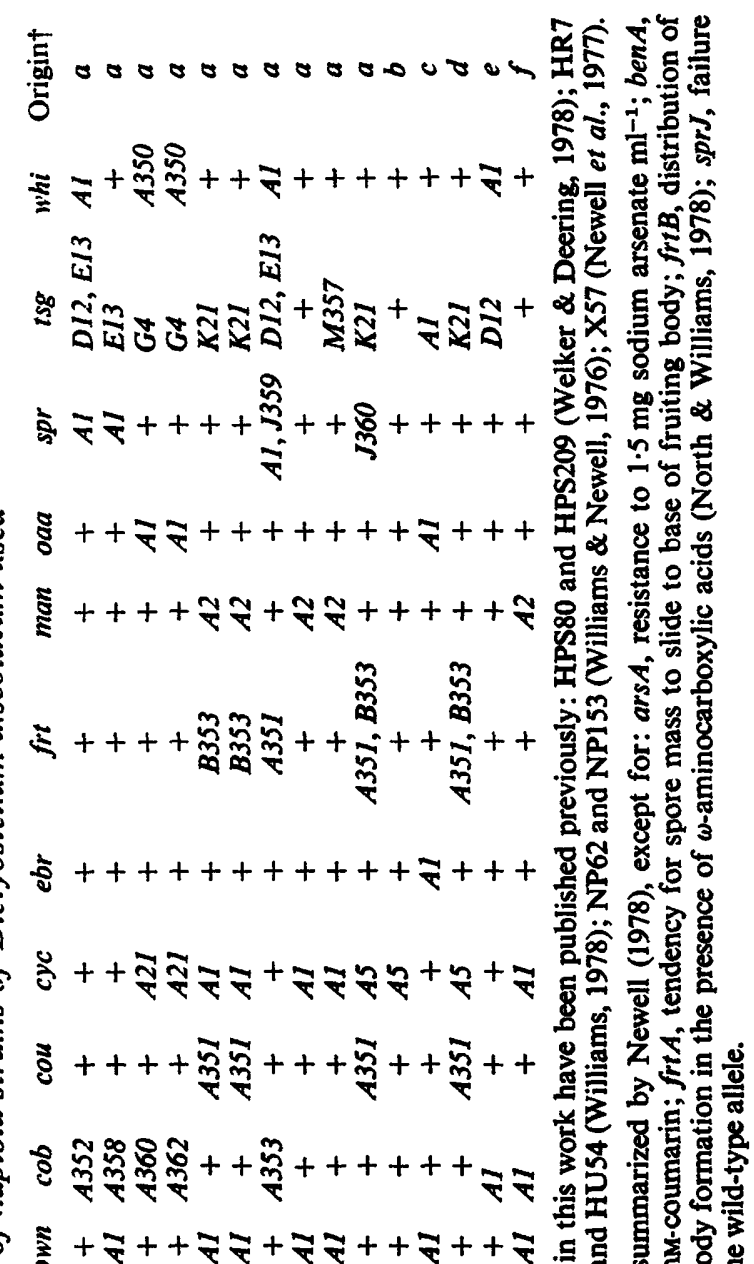

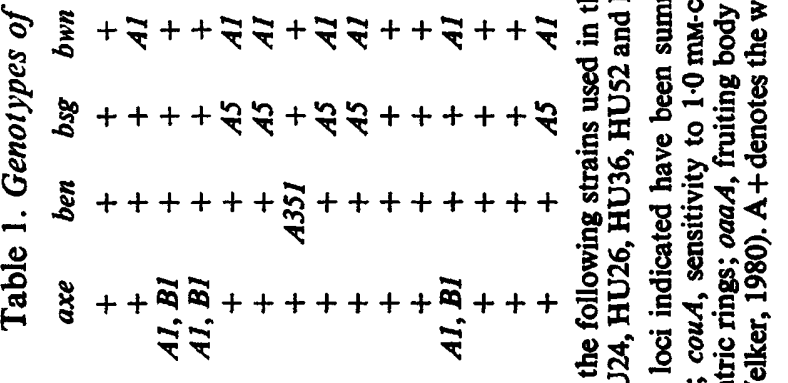

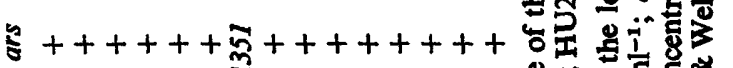

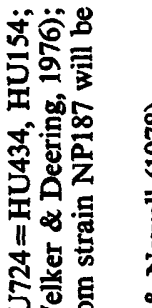
$\frac{\dot{0}}{2}$ a它

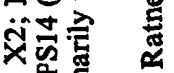
$\pm \mathbf{m}$.

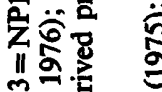

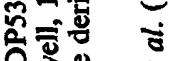

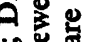
$\ddot{+} \bar{z}$ ॠे 空 5․ำ

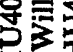
不 II $\tilde{x}$

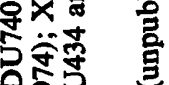
正垔 केष 会宊 实产岁 文言量

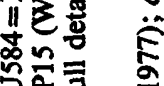
穴至

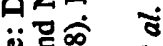
类

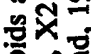
하월 品 焉め

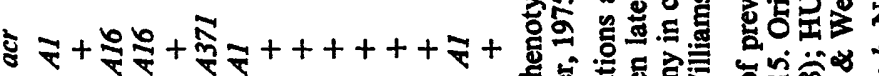
o 는

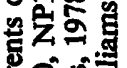

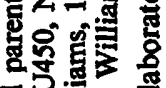

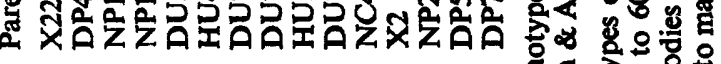

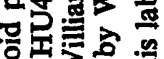

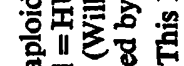

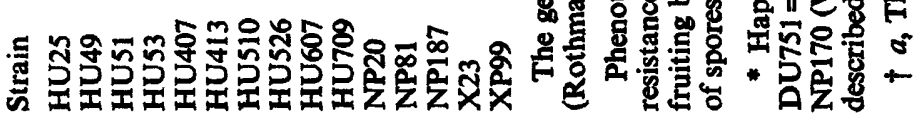




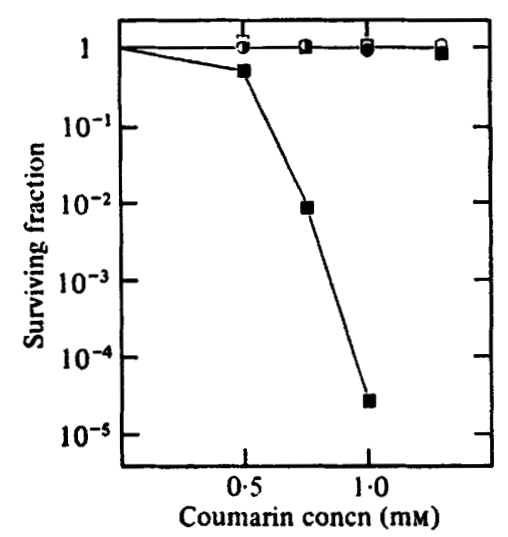

Fig. 1. Survival curves, on SM-agar containing between 0.5 and $1.3 \mathrm{~mm}$-coumarin, of the coumarinsensitive couA351 haploid NP187 (D) and two haploids having the wild-type level of coumarin resistance, NP20 (O), the parent of NP187, and XP99 (O). Also shown is the survival curve of DU584 ( ), a diploid heterozygous for the couA351 mutation which was constructed by fusion of haploids NP187 and XP99.

couA351 strain NP187 and strain XP99, had the same level of coumarin sensitivity as the wild-type haploids (Fig. 1).

The couA351 mutation was assigned to linkage group VII on the basis of its segregation relative to the cob $A 1$ and $t s g K 21$ mutations which have previously been assigned to linkage group VII (Williams \& Newell, 1976; Ratner \& Newell, 1978; Ross \& Newell, 1979). In haploids obtained from diploid DU584, the couA351 mutation always co-segregated with the tsgK21 mutation and never segregated with the $\operatorname{cob} A 1$ mutation (see Table 2). At the same time, it segregated independently of genetic markers on linkage groups III, IV and VI.

\section{Random haploidization by ben late}

Diploid DU584 was not marked on linkage groups I and II and, moreover, amongst the segregants of DU584 it was noted that only the parental combinations of linkage groups III and VI were present. Such linkage associations of particular linkage groups have been described previously (Welker \& Deering, 1978; Newell, 1978). To examine segregation with linkage groups I and II and to confirm that the association of linkage groups III and VI was not due to either the couA35I (coumarin sensitivity) mutation or to haploidization on ben late, we segregated on ben late another diploid, DU765, which was heterozygous for the couA351 mutation. As shown in Table 2 and in more detail in Table 3, where full genotypes of segregants are given, there was no co-segregation of particular linkage groups amongst the segregants of DU765. In fact, among the 105 haploid segregants obtained from DU765 we recovered 38 of the possible 64 classes of segregants identifiable with the genetic markers in DU765. Thus the linkage association seen amongst the segregants of DU584 cannot be due to either the couA351 mutation or the haploidization procedure. However, many segregant classes containing linkage group IV from strain HR7 were not represented. Presumably this is a result of deleterious mutation(s) on the linkage group IV of HR7, since segregation of linkage group IV from other diploids was random (Williams \& Barrand, 1978). The couA351 mutation segregated with the $t s g K 21$ mutation but independently of linkage groups I, II, III, IV and VI in the segregants of DU765, thereby confirming its assignment to linkage group VII. 


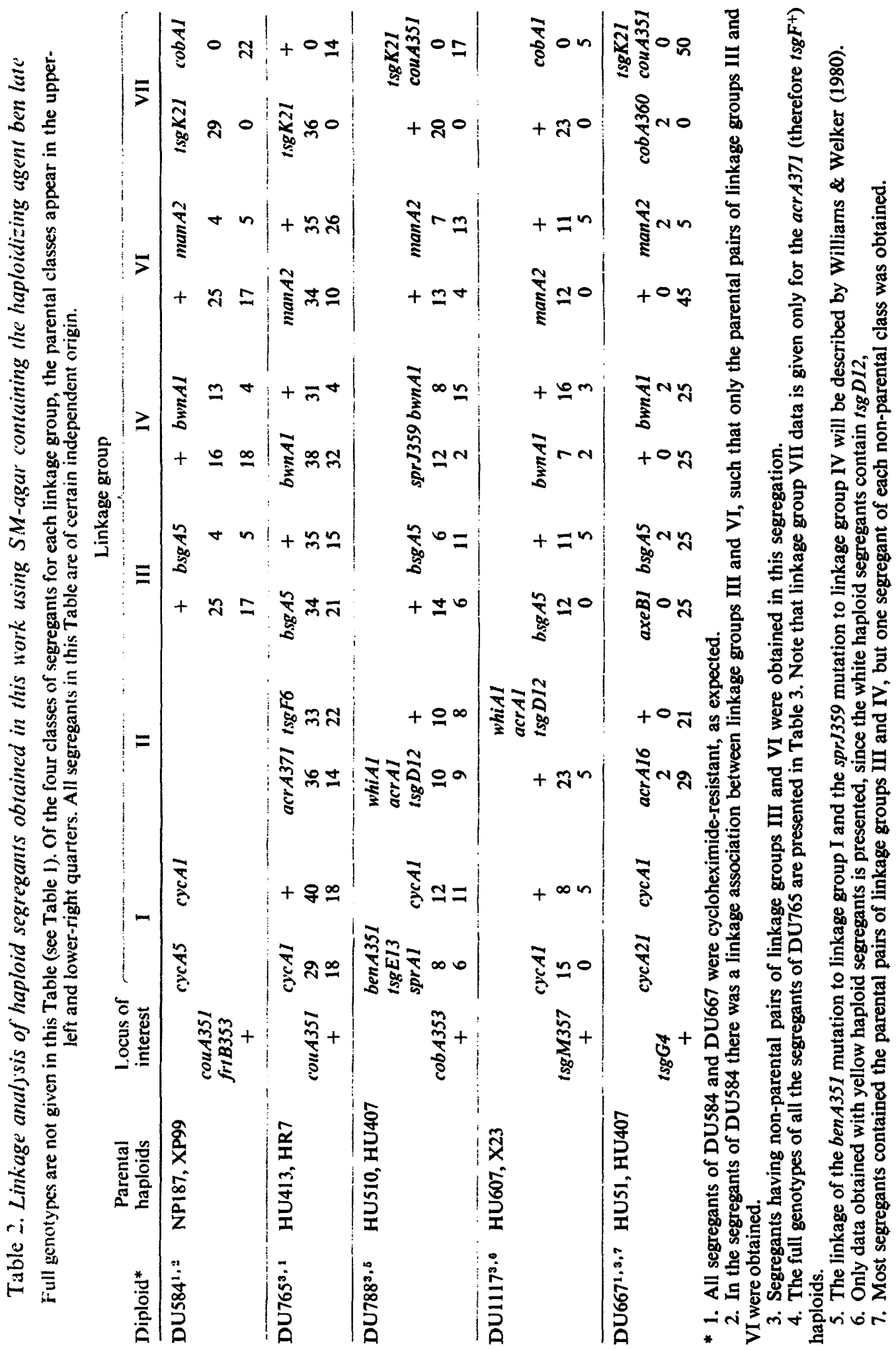


Table 3. The number of haploid segregants of DU765 of each identifiable class and their complete genotype

The 105 haploid segregants listed here are those described for DU765 in Table 2. All are of certain independent origin and were obtained by haploidization on ben late. The linkage group VII genotype of the acr $A^{+}$tsgF6 segregants was inferred from the presence or absence of the couA35I mutation.

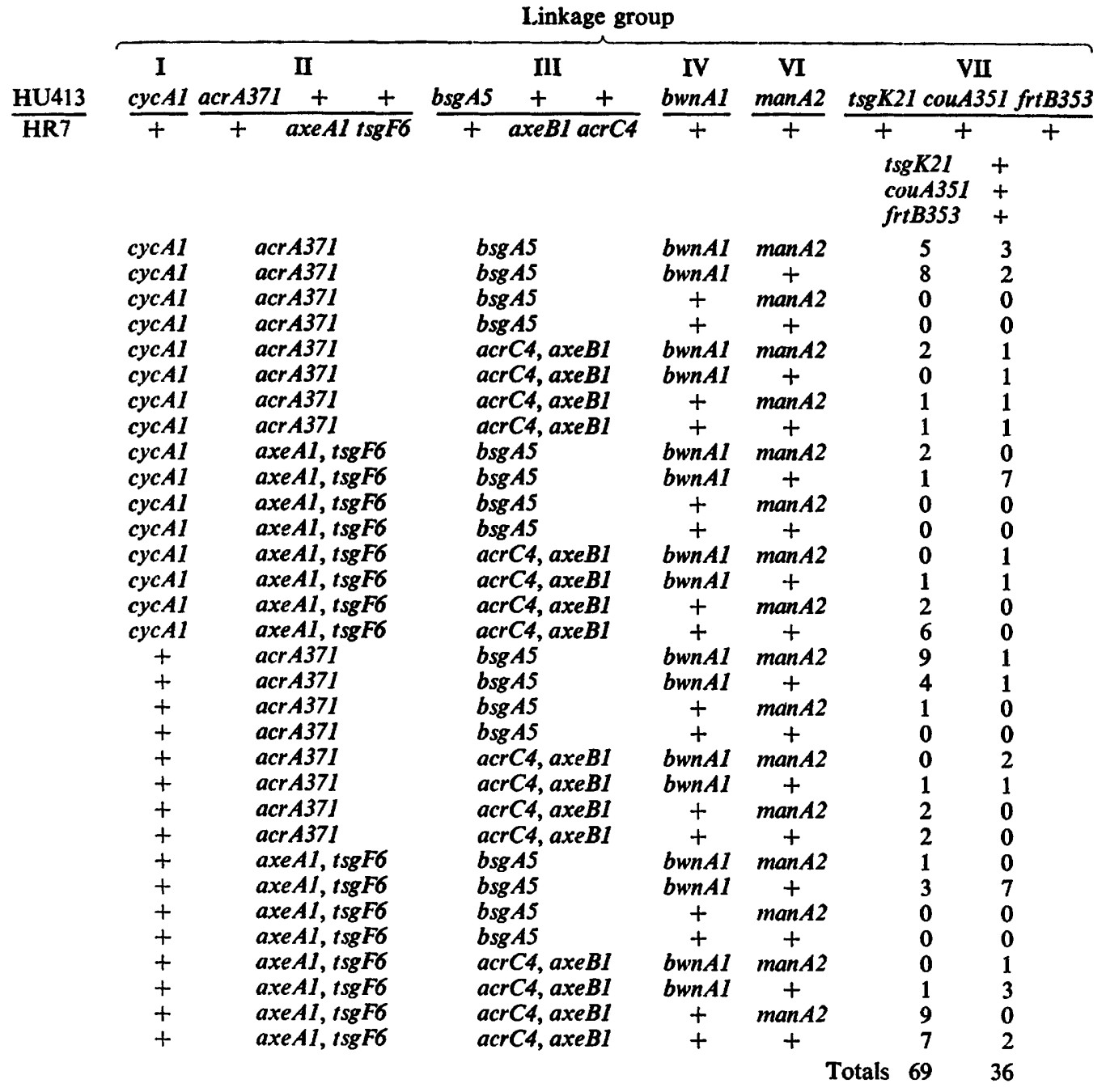

\section{A morphological mutation, frtB353, on linkage group VII}

In addition to the couA35I coumarin-sensitive mutation, strain NP187 was found to contain a mutation, frtB353, that affects the distribution of fruiting bodies in the slime mould colony. As shown in Fig. 2, the fruiting bodies of NP187 and HU709 (another frtB353 strain) appear more evenly spaced than those of NP20 (the parental strain of NP187) and tend to be formed in a series of concentric rings. This mutation was found to cosegregate with the $t s g K 21$ and couA351 mutations in the segregants of DU584 (Table 2) and of other diploids that contain linkage group VII from strain NP187. Thus the frtB353 mutation is also on linkage group VII. This mutation is not suitable as a standard marker as it is suppressed in some genetic backgrounds. 


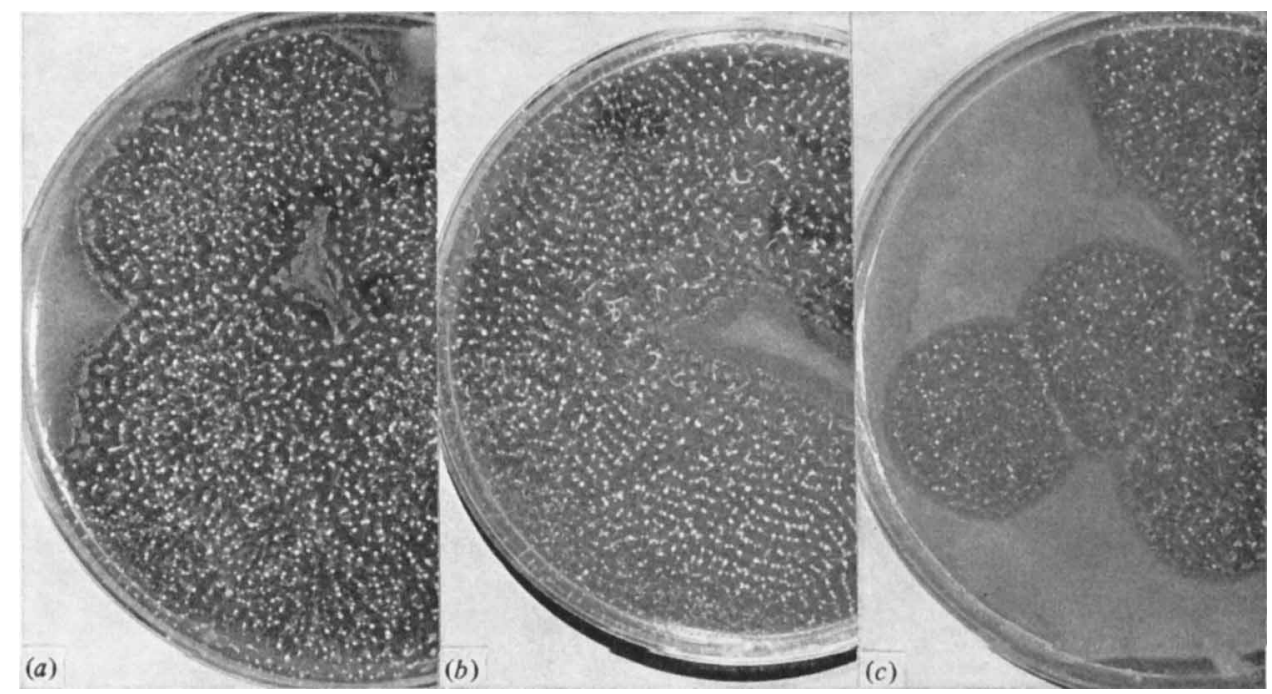

Fig. 2. The colony morphologies of two haploids bearing the frtB353 mutation, NP187 (a) and HU709 (b). Also shown is the colony morphology of haploid NP20 (c), the parent of NP187. Photographs were taken between 6 and $8 \mathrm{~d}$ after inoculation on $90 \mathrm{~mm}$ diam. SM-agar plates.

\section{Two additional temperature sensitivity mutations on linkage group VII}

A newly isolated temperature sensitivity mutation, $t s g M 357$, was assigned to linkage group VII on the basis of its segregation relative to the cobAl mutation in segregants of diploid DU1117 (Table 2). All yellow tsgM357 temperature-sensitive haploid segregants of DU1117 were unable to grow on SM-agar plates containing $300 \mu \mathrm{g} \mathrm{CoCl}_{2} \mathrm{ml}^{-1}$, while the yellow temperature-resistant segregants were able to grow under these conditions. The tsg M357 mutation also segregated independently of linkage groups I, II, III, IV and VI in the segregants of DU1117.

The temperature sensitivity mutation $t s g G 4$ was also shown to be on linkage group VII on the basis of its segregation relative to the cobA360, couA351 and tsgK21 mutations in the segregants of DU667 (Table 2). No temperature-resistant haploid segregants were obtained from DU667 although both cobalt-resistant and coumarin-sensitive haploid segregants were obtained as expected if the $t s g G 4$ mutation was on linkage group VII. Several other diploids constructed between haploids containing tsgG4 and $t s g K 21$ all failed to give temperatureresistant haploid segregants. All of six coumarin-sensitive, temperature-sensitive segregants of DU667 tested were able to form temperature-resistant diploids with HU52, a strain containing the tsgG4 mutation, while both of the cobalt-resistant, temperature-sensitive segregants of DU667 tested were able to form temperature-resistant diploids with HU413, a strain bearing the $t s g K 21$ mutation. Rothman \& Alexander (1975) have previously shown that $t s g G 4$ is not located on linkage groups I, II, III or IV. Our results are consistent with their finding and also indicate that the $t s g G 4$ mutation segregates independently of linkage group VI (Table 2). Further evidence for the assignment of tsgG4 to linkage group VII was obtained by the isolation of temperature-sensitive, cobalt-resistant diploids from DU667 (tsgG4 cobA360/+ + ; Table 2) which presumably arose via mitotic recombination. Three independent temperature-sensitive, cobalt-resistant diploids, and nine temperatureresistant, cobalt-resistant diploids which represent at least four independent recombination events, were found amongst $1.8 \times 10^{6}$ cells of DU667 inoculated on SM-agar plates containing $300 \mu \mathrm{g} \mathrm{CoCl}_{2} \mathrm{ml}^{-1}$. Therefore, the $t \mathrm{sg} G$ temperature sensitivity locus is on linkage group VII and is probably proximal to the $\operatorname{cob} A$ locus. 
Table 4. Dominance and complementation analysis of cob mutations

The cobA1, cobA351, cobA360, cobA361 and cobA363 alleles have previously been shown to be recessive to wild-type (Williams, 1978). The dominance of the cobA353 and cobA357 alleles is discussed in the text.

\begin{tabular}{|c|c|c|c|}
\hline Diploid & Haploid parents & cob mutations & $\begin{array}{l}\text { Characteristic of } \\
\text { diploid* }\end{array}$ \\
\hline $\begin{array}{l}\text { DU103 } \\
\text { DU491 }\end{array}$ & $\begin{array}{l}\text { HU25/NP81 } \\
\text { HU49/HPS80 }\end{array}$ & $\begin{array}{l}\text { cobA352, + } \\
\text { cobA358, + }\end{array}$ & $\begin{array}{l}\mathbf{S} \\
\mathbf{S}\end{array}$ \\
\hline DU199 & HU53/X57 & cobA362, + & $\mathbf{S}$ \\
\hline DU92 & NP62/X23 & $\operatorname{cob} A 1, \operatorname{cob} A 1$ & $\mathbf{R}$ \\
\hline DU145 & NP62/HU24 & $\operatorname{cob} A 1, \operatorname{cob} A 351$ & $\mathbf{R}$ \\
\hline DU144 & NP153/HU25 & $\operatorname{cob} A 1, \operatorname{cob} A 352$ & $\mathbf{R}$ \\
\hline DU1130 & XP99/HU26 & cobA1, cobA353 & $\mathbf{R}$ \\
\hline DU131 & X23/HU36 & $\operatorname{cob} A 1, \operatorname{cob} A 357$ & $\mathbf{R}$ \\
\hline DU1131 & XP99/HU51 & $\operatorname{cob} A 1, \operatorname{cob} A 360$ & \\
\hline DU1132 & XP99/HU53 & $\operatorname{cobA} 1, \operatorname{cob} A 362$ & $\mathbf{R}$ \\
\hline DU175 & HU24/HU52 & $\operatorname{cob} A 351, \operatorname{cob} A 361$ & $\mathbf{R}$ \\
\hline DU173 & HU49/HU51 & $\operatorname{cob} A 358, \operatorname{cob} A 360$ & $\mathbf{R}$ \\
\hline DU170 & HU49/HUS4 & cobA358, cobA363 & $\mathbf{R}$ \\
\hline
\end{tabular}

* S, Sensitive, denotes inability to grow on SM-agar containing 300 or $350 \mu \mathrm{g} \mathrm{CoCl}_{2} \mathrm{ml}^{-1}$ although resistant haploids and diploids are observed as a result of haploidization and mitotic crossing-over, respectively. $R$, Resistant, denotes ability to grow on SM-agar containing 300 or $350 \mu \mathrm{g} \mathrm{CoCl}_{2} \mathrm{ml}^{-1}$.

\section{Complementation tests between cobalt-resistant mutants - a single cob locus}

A series of cobalt-resistant mutants were isolated recently (Williams, 1978) but not mapped. Here nine new $c o b$ mutations are assigned to $\operatorname{cob} A$ by complementation analysis, and in the case of cob-353, its location on linkage group VII is confirmed by analysis of segregants from a diploid heterozygous for $t s g K 21$, couA351 and cob-353 (Table 2). Complementation tests between cobalt-resistant mutants are not as straightforward as such tests between, say, cyc mutants, since SM-agar plates containing $\mathrm{CoCl}_{2}$ are not uniformly toxic (Williams, 1978). However, by doing appropriate controls and examining clonal platings of the diploids, all the available evidence is consistent with the ten $c o b$ mutations studied here (Table 4) mapping at the single locus which has previously been designated $\operatorname{cob} A$ (Williams \& Newell, 1976; Ratner \& Newell, 1978). In some cases, several diploids were constructed using different combinations of mutations to cover possible genetic background effects. The alleles showing dominance to wild-type required special study with SM-agar containing $\mathrm{CoCl}_{2}$ at concentrations ranging from 300 to $400 \mu \mathrm{g} \mathrm{ml}^{-1}$. In particular, the $\operatorname{cob} A 353$ allele was studied in over 50 diploids to establish its level of resistance and dominance. Diploids heterozygous for cobA353 are usually fully resistant at the edge of an SM-agar plate containing $350 \mu \mathrm{g} \mathrm{CoCl}_{2} \mathrm{ml}^{-1}$, but show poor growth in the centre. Diploids carrying both cobAI and cobA353 (e.g. DU1130, Table 4) showed greater resistance in that growth was good over the whole plate. Thus cobA353 has been scored as an allele of $c o b A$. Similar results were found with the $\operatorname{cob} A 357$ allele which is incompletely dominant to wild-type (Williams, 1978). Strain HU32, which carries cob-354, a cobaltous resistance mutation requiring passage in the presence of $\mathrm{CoCl}_{2}$ for maintenance of the resistance phenotype (Williams, 1978), was not studied here.

The linkage data is therefore consistent with the notion that all $c o b$ alleles studied here map at $\operatorname{cob} A$ on linkage group VII. In particular, the $\operatorname{cob} A 353$ mutation is clearly located on linkage group VII on the basis of its independent segregation from markers on linkage groups I, II, III, IV and VI and its segregation relative to the tsgK12 and couA35I linkage group VII markers amongst the segregants of DU788 (Table 2). 


\section{Use of coumarin sensitivity in selection of heterozygous diploids}

We showed that coumarin sensitivity mutations could be used in the selection of heterozygous diploids by crossing the couA351-containing haploid strain NP187 with HPS209, a haploid strain bearing the bsgA5 mutation. After allowing fusion of the haploids to occur, approximately $10^{6}$ cells were inoculated in association with Bacillus subtilis to each of five SM-agar plates and five SM-agar plates containing $1.0 \mathrm{mM}$-coumarin. The SM-agar plates were incubated at $26.5 \pm 0.2{ }^{\circ} \mathrm{C}$ to isolate diploids on the basis of complementation of the bsg $A 5$ and $t s g K 2 I$ mutations, as described by Newell et al. (1977), while the SM-agar plates containing $1.0 \mathrm{~mm}$-coumarin were incubated at $21 \pm 1{ }^{\circ} \mathrm{C}$ to select diploids on the basis of complementation of the couA35I and bsg A5 mutations. After 6 and $9 \mathrm{~d}$, respectively, colonies appearing on the SM-agar plates and SM-agar plates containing coumarin were picked to SM-agar plates spread with $K$. aerogenes to allow better development. The ploidy of the resulting colonies was determined on the basis of spore size. We obtained frequencies of diploid colonies of $1.7 \times 10^{-5}$ from the plates incubated at $26.5 \pm 0.2{ }^{\circ} \mathrm{C}$ and $1.4 \times 10^{-5}$ from the plates containing coumarin which were incubated at $21 \pm 1^{\circ} \mathrm{C}$. Thus complementation of coumarin sensitivity mutations is an effective method of selecting heterozygous diploids that result from the fusion of pairs of haploids and gives diploids at frequencies similar to those obtained using other selective techniques.

\section{DISCUSSION}

Only four loci ( $\operatorname{cob} A, \operatorname{tsg} K, \operatorname{stm} A$ and $\operatorname{stm} F$; mutations at the $\operatorname{stm}$ loci lead to large aggregation streams) have been previously described for linkage group VII (Williams \& Newell, 1976; Ratner \& Newell, 1978; Ross \& Newell, 1979) and only two of these (cobA and $t s g K)$ are suitable as markers for routine genetic analysis. Mutations at three of the four additional loci (couA, frtB, tsgM and tsgG) on linkage group VII which we describe (tsgM357, tsgG4 and, in particular, the couA35I mutation) should be valuable markers for genetic analysis of $D$. discoideum. The assignment of $t s g G$ to linkage group VII resolves the uncertainty about the location of this locus. Rothman \& Alexander (1975) previously established that t. $\mathrm{gg}$ was not located on linkage groups I, II, III or IV. The mapping of nine more cobalt resistance mutations, including the dominant mutation cobA353, to the cobA locus on linkage group VII makes available a number of different alleles at $c o b A$ and is also consistent with there being only a single locus for high levels of resistance to $\mathrm{CoCl}_{2}$ in $D$. discoideum.

While the possibility exists that the phenotypes suggested for the $\operatorname{tsg} K 21$, couA351 and frt 3353 mutations are the pleiotropic effects of a single mutation, our current data are inconsistent with this hypothesis. It is clear that the frtB353 phenotype is suppressible in some genetic backgrounds yet such strains remain temperature-sensitive and coumarinsensitive. We have characterized strains in which the couA351 mutation is partially suppressed yet the $t s g K 21$ mutation remains fully expressed. Thus, it is probable that the $t s g K 21$, couA35l and frtB353 mutations are an example of multiple mutations induced by MNNG. Further examples of multiply mutated strains isolated following mutagenesis with MNNG are described by Williams \& Newell (1976) and Rothman \& Alexander (1975).

The segregation results presented here highlight a problem with parasexual linkage analysis in $D$. discoideum. It is clear that in certain genotypes all linkage groups do not segregate freely but combinations of parental linkage groups co-segregate. The possibility that this aberrant segregation is due to failure to examine independently derived haploid segregants is excluded here, since all segregations were done using ben late which gives only independently derived haploids (Williams \& Barrand, 1978). This co-segregation is particularly striking in the case of linkage groups III and VI in DU584 (Table 2). Until this problem 
is resolved it is important to provide evidence for independent segregation of other linkage groups as well as co-segregation with a particular linkage group before a new locus is assigned to a linkage group. Several possible explanations for such co-segregation are being examined, including translocations (Welker \& Deering, 1978) and chromosome fusion (D. L. Welker \& K. L. Williams, unpublished results). It seems likely that at least some cosegregations have a different genetic basis. For example, we are examining the segregation of linkage groups III and VI in diploids of different genotype and it seems possible that the factor(s) involved may reside on linkage group(s) other than linkage group III or VI.

The coumarin sensitivity mutation couA351 is useful both as a genetic marker and in the selection of diploid strains. Previous selective procedures for the isolation of diploids following fusion of pairs of parental haploid cells have, for the most part, relied on the use of recessive temperature sensitivity mutations affecting growth in at least one haploid parent (Loomis, 1969; Welker \& Deering, 1976; Newell et al., 1977; Williams, 1978) or mutations deleterious to growth under normal conditions (Loomis \& Ashworth, 1968). The use of strains that bear mutations affecting vegetative growth, either at the permissive or restrictive growth temperature, is undesirable when characterizing the effects of temperature on developmental processes or in the study of strains with temperature-sensitive mutations that specifically affect development. The use of other sensitivity mutations provides a means of selecting diploids that do not require the presence of temperature sensitivity mutations in the parental haploids. Newell et al. (1977) discovered the bsg $A$ locus which results in inability to grow on $B$. subtilis and the bsg $A 5$ mutation has been extremely useful. Drug sensitivity is another possible source of sensitivity mutations. Previous reports have described briefly the use of methanol and cycloheximide sensitivity mutations in diploid selection (Newell et al., 1977; Williams, 1978). However, as pointed out in those reports, cycloheximide and methanol resistance mutations are already present in many of the strains most useful in genetic studies, and thus methanol and cycloheximide sensitivity mutations are of limited value in diploid selection. Coumarin sensitivity mutations such as couA351 do not have this drawback, since no mutations leading to enhanced resistance to coumarin have been reported in $D$. discoideum. Therefore, coumarin sensitivity mutations are potentially as useful as bsg mutations for diploid selection in $D$. discoideum and we are characterizing several new coumarin sensitive mutants.

We wish to thank A. Chambers, R. Smith and N. Fitzpatrick for expert technical assistance. We also wish to thank Dr P. C. Newell for the gift of strain NP187. D. L. W. was supported by Post-doctoral Fellowship Grant DRG-206-F, from the Damon RunyonWalter Winchell Cancer Fund and a U.S. National Institutes of Health Post-doctoral Fellowship from the National Cancer Institute (1 F32 CA06162-01).

\section{REFERENCES}

Free, S. J., SChimke, R. T. \& LoOmis, W. F. (1976). The structural gene for $\alpha$-mannosidase-1 in Dictyostelium discoideum. Genetics 84, 159-174.

Looms, W. F. (1969). Temperature-sensitive mutants of Dictyostelium discoideum. Journal of Bacteriology 99, 65-69.

Looms, W. F. (1975). Dictyostelium discoideum : A Developmental System. New York: Academic Press.

Loomis, W. F. \& Ashworth, J. M. (1968). Plaquesize mutants of the cellular slime mould Dictyostelium discoideum. Journal of General Microbiology 53, 181-186.

Mosses, D., Williams, K. L. \& Newell, P. C. (1975). The use of mitotic crossing-over for genetic analysis in Dictyostelium discoideum: mapping of linkage group II. Journal of General Microbiology 90, 247-259.

NEWELL, P. C. (1978). Genetics of the cellular slime moulds. Annual Review of Genetics 12, 69-93.

Newelu, P. C., Henderson, R. F., Mosses, D. \& RATNER, D. I. (1977). Sensitivity to Bacillus subtilis: a novel system for selection of heterozygous diploids of Dictyostelium discoideum. Journal of General Microbiology 100, 207-211.

NoRTH, M. J. \& Williams, K. L. (1978). Relationship between the axenic phenotype and sensitivity to $\omega$-amino carboxylic acids in Dictyostelium discoideum. Journal of General Microbiology 107, 223-230. 
Ratner, D. I. \& Newell, P. C. (1978). Linkage analysis in Dictyostelium discoideum using multiply marked tester strains: establishment of linkage group VII and the reassessment of earlier linkage data. Journal of General Microbiology 109, 225-236.

Robson, G. E. \& Williams, K. L. (1977). The mitotic chromosomes of the cellular slime mould Dictyostelium discoideum: a karyotype based on Giemsa banding. Journal of General Microbiology 99, 191-200.

Ross, F. M. \& Newell, P. C. (1979). Genetics of aggregation pattern mutations in the cellular slime mould Dictyostelium discoideum. Journal of General Microbiology 115, 289-300.

Rothman, F. G. \& Alexander, E. T. (1975). Parasexual genetic analysis of the cellular slime mold Dictyostelium discoideum A3. Genetics 80, 715-731.

Sussman, M. (1966). Biochemical and genetic methods in the study of cellular slime mold development. Methods in Cell Physiology 2, 397410.

Welker, D. L. \& Deering, R. A. (1976). Genetic analysis of radiation-sensitive mutations in the slime mould Dictyostelium discoideum. Journal of General Microbiology 97, 1-10.

Welker, D. L. \& DeERING, R. A. (1978). Genetics of radiation sensitivity in the slime mould Dictyostelium discoideum. Journal of General Microbiology 109, 11-23.
WILliams, K. L. (1978). Characterization of dominant resistance to cobalt chloride in Dictyostelium discoideum and its use in parasexual genetic analysis. Genetics $90,37-47$.

Williams, K. L. \& Barrand, P. (1978). Parasexual genetics in the cellular slime mould Dictyostelium discoideum: haploidisation of diploid strains using ben late. FEMS Microbiology Letters 4, 155-159.

Williams, K. L. \& Newell, P. C. (1976). A genetic study of aggregation in the cellular slime mould Dictyostelium discoideum using complementation analysis. Genetics 82, 287-307.

Williams, K. L. \& Welker, D. L. (1980). Developmental Genetics (in the Press).

Williams, K. L., Kessin, R. H. \& Newell, P. C. (1974). Parasexual genetics in Dictyostelium discoideum: mitotic analysis of acriflavin resistance and growth in axenic medium. Journal of General Microbiology 84, 59-69.

Wright, M. D., Williams. K. L. \& Newell, P. C. (1977). Ethidium bromide resistance, a selective marker located on linkage group IV of Dictyostelium discoideum. Journal of General Microbiology 102, 423-426.

ZaDA-Hames, I. M. (1977). Analysis of karyotype and ploidy of Dictyostelium discoideum using colchicine-induced metaphase arrest. Journal of General Microbiology 99, 201-208. 